\title{
Light propagation in a photonic crystal fiber infiltrated with mesogenic azobenzene dyes
}

\author{
Daniel Budaszewski and Tomasz R. Woliński \\ Faculty of Physics, Warsaw University of Technology, Koszykowa 75, 00-662 Warszawa, Poland
}

Received April 20, 2017; accepted June 30, 2017; published June 30, 2017

\begin{abstract}
In this paper, light propagation has been investigated in an isotropic photonic crystal fiber as well in a silica-glass microcapillary infiltrated with a mesogenic azobenzene dye. It appeared that the light spectrum guided inside the photonic crystal fiber infiltrated with the investigated azobenzene dye depends on the illuminating wavelength of the absorption band and on linear polarization. Also, the alignment has been investigated of the mesogenic azobenzene dye molecules inside silica glass microcapillaries and photonic crystal fibers. The results obtained may lead to a new design of optically tunable photonic devices.
\end{abstract}

Over the last years, photonic crystal fibers (PCFs) have become the object of extensive studies by many research groups, mainly due to their outstanding optical properties [1]. The structure of PCFs consists of a periodic matrix of micro holes forming a 2-dimmensional photonic crystal with a defect in the core region [2]. The light guided inside PCFs can be dynamically modified by infiltrating the microchannels with materials whose refractive indices are sensitive to external physical factors as e.g. electric and magnetic fields or temperature. Amongst many potential materials to be introduced into PCFs micro holes, liquid crystals (LCs) seem to be the most suitable due to their electro-optical properties.

Such a PCF combination infiltrated with an organic guest liquid crystal is known as a photonic liquid crystal fiber (PLCF) [3-7]. The vast majority of research efforts in this area have been so far devoted to PCFs infiltrated with nematic LCs. Also, some progress has been reported in investigations of PCFs infiltrated either with chiral nematics [8] or type $\mathrm{C}$ chiral smectics that possess ferroelectric properties [9]. One of the most important challenges related to PLCFs is a proper alignment of LC molecules inside cylindrical microstructures, which are generally aligned homogeneously by the flow (in the case of nematic LCs). However, in order to obtain any desired alignment of LC molecules, it is necessary to deposit aligning materials on the boundary surfaces [10]. As it was previously reported, by covering the inner sides of a silica-glass microcapillary with azo-dye SD1 (DIC, Japan) illuminated with a polarized UV light beam it was possible to align - in a controlled way - both nematic LC molecules [11] and chiral smectic C LC molecules [12]. However, to change the alignment of LC molecules, it was necessary to illuminate the sample with a UV light beam with a different azimuth of polarization. Another method of aligning LC molecules inside cylindrical structures is based on the infiltration of a photonic structure with a mixture of pure LC doped with a photosensitive azo-dye [13-14] that is characterized by the cis-trans isomerization mechanism dependent on the applied wavelength [15].

In this work, we have initially examined the alignment of mesogenic azobenzene dye 5721 (BEAM C.O.) [16] inside a silica-glass microcapillary with an inner diameter of 6 microns.

The 5721 azo-dye LC is a multi-component compound based on a series of 4-n-alkyl-4'-n-alkoxy azobenzenes. Some of its electro-optical parameters are presented in Table 1.

Table 1. Electro-optical parameters of 5721 azo-dye, where birefringence $\Delta \mathrm{n}$ is given for $\lambda=632 \mathrm{~nm}, \mathrm{n}_{2}$ is the nonlinear index of refraction measured for $\lambda=532 \mathrm{~nm}$ and for light intensity $\mathrm{I}=4.4 \cdot 10^{-}$ ${ }^{7} \mathrm{~W} / \mathrm{cm}^{2}$. Both values of dielectric susceptibility were given for a frequency of $1 \mathrm{kHz}$. $\mathrm{U}_{\mathrm{F}}$ stands for the Fredericks transition threshold voltage.

\begin{tabular}{|c|c|c|c|c|c|}
\hline $\mathrm{T}\left[{ }^{\circ} \mathrm{C}\right]$ & $\begin{array}{c}\Delta \mathrm{n} \\
(@ 632 \mathrm{~nm})\end{array}$ & $\begin{array}{c}\mathrm{n}_{2} \\
{\left[\mathrm{~cm}^{2} / \mathrm{W}\right]}\end{array}$ & $\varepsilon_{\perp}$ & $\varepsilon_{\|}$ & $\mathrm{U}_{\mathrm{F}}[\mathrm{V}]$ \\
\hline $8 \mathrm{~N} 70 \mathrm{I}$ & 0.23 & 0.21 & 2.6 & 3.3 & 15 \\
\hline
\end{tabular}

Additionally, we have measured the refractive indices of the 5721 azo-dye LC in a trans- and cis- state at $\lambda=632 \mathrm{~nm}$ by using the wedge cell method [17]. In a trans-state, the ordinary and extraordinary refractive indices were 1.55 and 1.78, respectively. For a cis-state when the investigated material is isotropic, the refractive index was equal to 1.59 .

After infiltration, a microcapillary sample was exposed for 5 minutes to the linearly polarized light from a high power electroluminescent diode (LED) emitting at $\lambda=450 \mathrm{~nm}$ with the polarization direction perpendicular to the microcapillary axis. The sample was then examined under a microscope with crossed polarizers and a red filter in order to avoid any unpredicted isomerisation of azo-dye molecules caused by white light. The results have been shown in Fig. 1. 

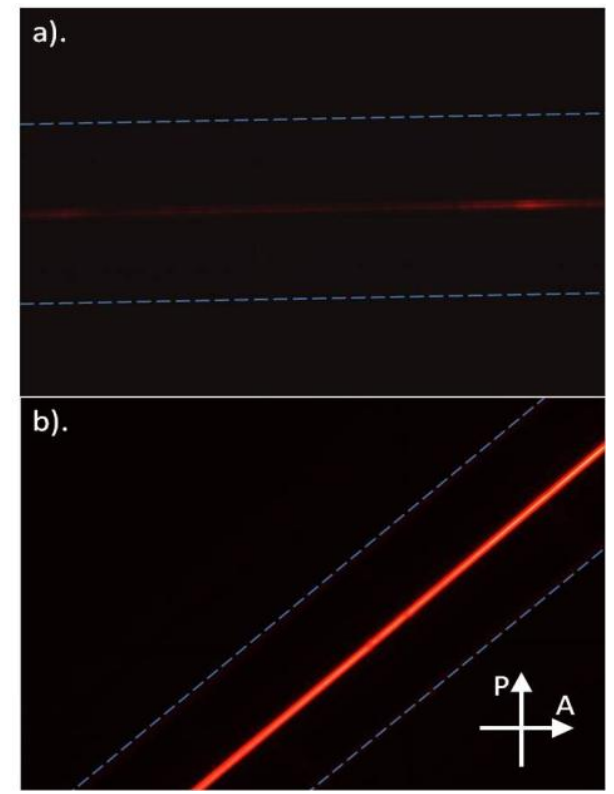

Fig. 1. A 6-micron microcapillary infiltrated with mesogenic azo-dye 5721 (BEAM CO) observed under a polarizing microscope with an additional red filter. (a) the sample was parallel and (b) at 45 degrees to the analyzer's polarization axis.

When the azimuth between the investigated sample and the crossed polarizers was 0 degrees in the infiltrated part of the sample, a dark region was observed that indicates a planar alignment of LC molecules along the sample axis (Fig. 1a). Furthermore, when the azimuth between the crossed polarizers and the sample was 45 degrees, the molecules were also at 45 degrees and hence a higher intensity of the light passing through the sample was observed (Fig. 1b).

The investigated sample was next illuminated with a UV LED light beam $(\lambda=405 \mathrm{~nm})$. As a result, cisphotoisomerization occurred and the molecular structure of the mesogenic material changed into an isotropic state.

In the next step of our research, an isotropic PCF LMA-10 (NKT Photonics) was infiltrated with 5721 azodye on the $1 \mathrm{~cm}$ section. The infiltrated PCF was coupled with a multimode fiber by using UV curable glue. Experimental measurements were performed by using the setup shown in Fig. 2.

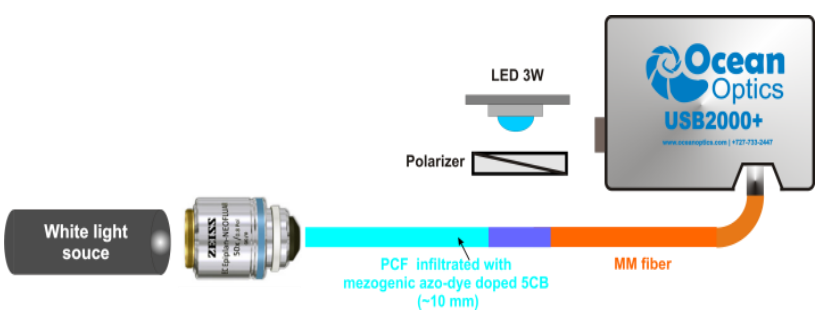

Fig. 2. Experimental setup
The light from a halogen lamp (Mikropack HL-2000) was coupled into the investigated PLCF and was registered by a fiber optic spectrometer (Ocean Optics HR-2000). The infiltrated part of the sample was illuminated by UV or blue high-power LEDs to induce cis- or trans-isomerization of the azo-dye molecules inside the investigated PLCF sample. The results are shown in Fig. 3.
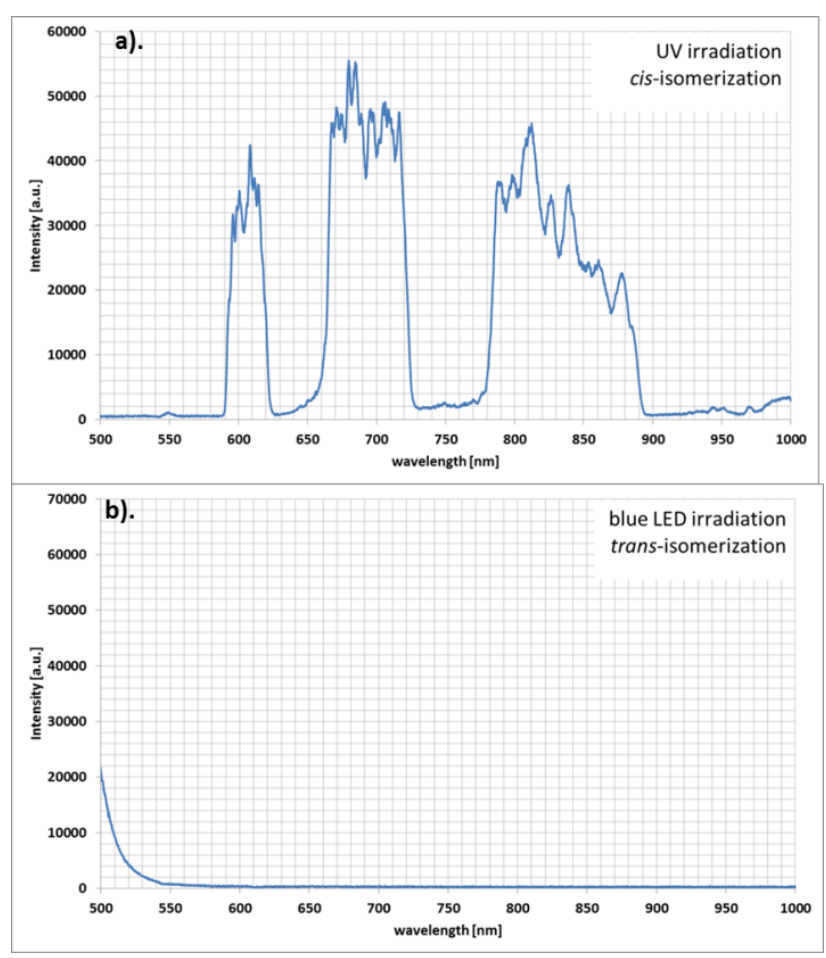

Fig. 3. Light spectrum guided in LMA-10 PCF infiltrated with azo-dye 5721 after illumination with a UV LED (a) and a blue LED (b).

When the investigated sample was illuminated with a UV LED, light propagation governed by a photonic bandgap effect was observed. Nevertheless, when the sample was illuminated with a linearly polarized blue LED, the propagation of light faded completely. One of the possible explanations for this phenomenon might be that the polarization direction of a blue LED light beam must have been distorted by the complex structure of the PCF. As a result of trans-photoisomerization, the azo-dye molecules alignment was random, which enhanced the scattering losses of the light guided in the sample.

In order to verify this hypothesis we have prepared a solution of 5721 azo-dye with 5CB nematic LC with a concentration of azo-dye in this solution $\sim 5 \%$ in volume. Another section of the LMA-10 PCF was infiltrated with the prepared mixture and measured in the setup presented in Fig. 2. As it was in the previous PLCF sample, the infiltrated part was $1-\mathrm{cm}-$ long. The results are presented in Fig. 4. 

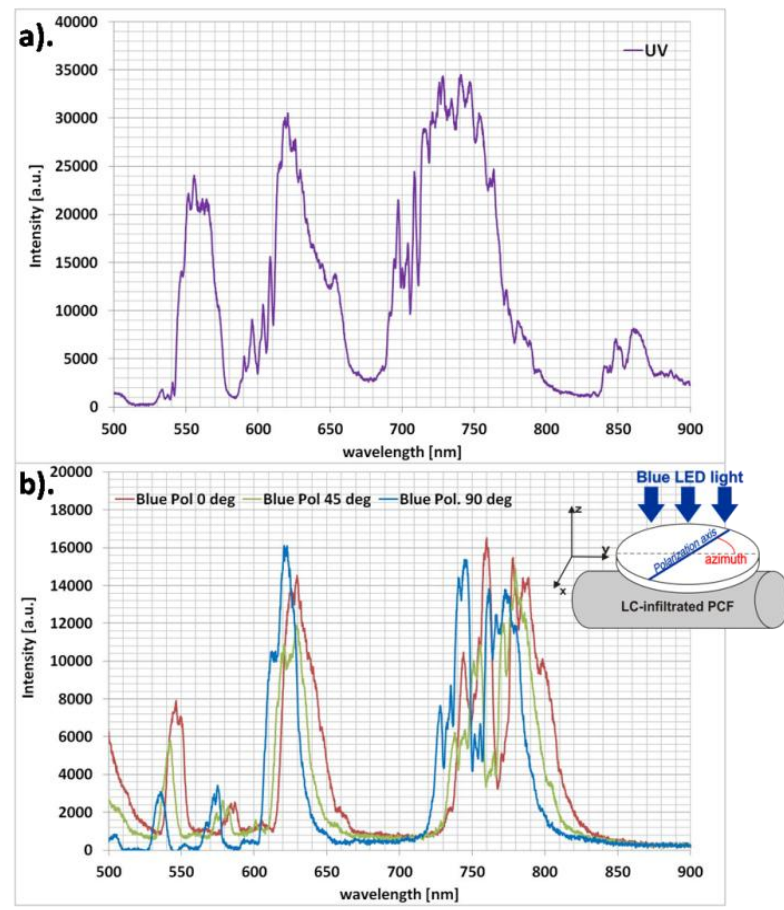

Fig. 4. Spectra of the halogen light guided in LMA10 PCF infiltrated with 5721 azo-dye with 5CB sample for a UV light (a) and a linearly polarized blue light (b) with different azimuths between the light polarization and the optical axis of the sample.

After illumination of the infiltrated part of the LMA10 PCF infiltrated with a mixture of 5721 azo-dye and 5CB with a UV LED light beam we have observed light propagation based on a photonic bandgap effect (Fig. 4a). In comparison to the results from Fig. 3a, the bandgaps were blue-shifted. The observed blue-shift must be caused by different effective refractive indices of both systems.

Next, the investigated PLCF sample was illuminated with a linearly polarized blue LED for azimuths 0,45 , and 90 degrees in respect to the PLCF's optical axis.

The observed spectrum of the light guided in the PLCF sample was blue-shifted depending on the azimuth of light polarization (Fig. 4b). The observed shift of the bandgaps was about $14 \mathrm{~nm}$. Moreover, during illumination of both investigated PLCF samples with LEDs it was observed that the time needed to conduct photoisomerization was shorter for the PLCF infiltrated with 5721 azo-dye than for the PLCF infiltrated with a mixture of azo-dye and 5CB. It should be mentioned that switching times depends also on the power density of an illuminating LED beam. The power density of LED sources used in the experiment was $0.66 \mathrm{~mW} / \mathrm{cm}^{2}$ for a UV LED and $2.2 \mathrm{~mW} / \mathrm{cm}^{2}$ for a blue LED. These values are rather weak, and that is why the obtained switching times were not very short. In the case of a PLCF with pure azo-dye, the switching time from a trans- into cisstate took $\sim 30$ seconds. For photoisomerization from a cis- to trans- state the switching time was similar.
However, for PLCF infiltrated with a mixture of azo-dye and $5 \mathrm{CB}$, the switching times of photoisomerization were $\sim 90$ seconds.

It can be explained that for samples where azo-dye materials serve as a dopant in liquid crystals, only a small amount of molecules take part in the photoisomerization process [18]. It might suggest that the photoisomerization process is not directly responsible for the optical properties of the sample. LC molecules adapt to a new alignment of the dopant, which is rather a slow process in comparison to samples infiltrated only with a mesogenic azo-dye LC. In this case, all the azo-dye molecules can take part in the photoisomerization process and as a result, the reorientation of molecules can be achieved in a very short time [19]. In our experiment, the switching times could be minimized by illuminating with light sources with higher power density e.g. laser diodes.

Further research agenda in this field will include the application of metallic nanoparticles to enhance the electro-optical parameters of a PLCF.

This work was partially supported by the National Science Centre under the grant No. 2015/19/B/ST7/03650 and partially by the COST Action Program IC1208

\section{References}

[1] P. Russell. St. J. J. Lightwave Technol. 24, 4729 (2006)

[2] T. Larsen, A. Bjarklev, D. Hermann, J. Broeng, Opt. Exp. 11, 2589 (2003).

[3] D.C. Zografopoulos, A. Asquini, E.E. Kriezis, A. d'Alessandro, R. Beccherelli, Lab Chip, 12, 3598 (2012).

[4] F. Du, Y-Q. Lu, S-T. Wu, Appl. Phys. Lett 85, 2181 (2004).

[5] D.C. Zografopoulos, E.E. Kriezis, J. Lightwave Technol. 27(6), 773 (2009).

[6] S. Ertman, M. Tefelska, M. Chychłowski, A. Rodriquez, D. Pysz, R. Buczyński, E. Nowinowski-Kruszelnicki, R. Dąbrowski, T.R. Woliński, J. Lightwave Technol. 30, 1208 (2012).

[7] D. Noordegraaf, L. Scolari, J. Laegsgaard, L. Rindorf, T.T. Alkeskjold, Opt. Expr. 15, 7901 (2007).

[8] M.M. Tefelska, M.S. Chychlowski, T.R. Wolinski, R. Dabrowski, W. Rejmer, E. Nowinowski-Kruszelnicki, P. Mergo, Mol. Cryst. Liq Cryst. 558(1), 184 (2012).

[9] S. Mathews, Y. Semenova, G. Farrell, Electr. Lett. 45(12), 617 (2009)

[10] V. Chigrinov, H.-S. Kwok, H. Takada, H. Takatsu, Liquid Crystals Today, 14:4, 1 (2005).

[11] A. Siarkowska, M. Jóźwik, S. Ertman, T.R. Woliński, V.G. Chigrinov, Opto-Electon. Rev. 22(3), 178 (2014).

[12] D. Budaszewski, A.K. Srivastava, A.M.W. Tam, T.R. Woliński, V.G. Chigrinov, H.-S. Kwok, Opt. Lett. 39, 16 (2014).

[13] J.-H. Liou, T.-H. Chang, T. Lin, Ch.-P. Yu, Opt. Expr. 19(7), 6756 (2011).

[14] T.T. Alkeskjold, J. Laegsgaard, A. Bjarklev, D.S. Hermann, J. Broeng, J. Li, S-T. Wu, Opt. Exp, 12(24), 5857 (2004).

[15] K. Ichimura, Y. Suzuki, T. Seki, A. Hosoki, K. Aoki, Langmuir 4, 1214 (1988).

[16] http://www.beamco.com/Azobenzene-liquid-crystals

[17] K.A. Rutkowska, K. Orzechowski, M. Sierakowski, Phot. Lett. Poland, 8(2), 51 (2016).

[18] L. Deng, H.-K. Liu, Opt. Eng. 42, 2936 (2003).

[19] J. Si, J. Qiu, J. Guo, M. Wang, K. Hirao, Appl. Opt. 42, 7170 (2008). 